\title{
L'homme et la ville, approche systémique de l'urbanisme par le neurobiologiste Henri Laborit
}

\author{
Cyrille Harpet \\ Philosophe et anthropologue, EHESP, département Santé environnement travail et génie sanitaire, 35043 Rennes, France
}

Mots-clés :

urbanisme; approche systémique ; systèmes complexes ; comportements sociaux ; neurobiologie

\begin{abstract}
Résumé - Henri Laborit (1914-1995) est un neurochirurgien ayant mis au point les premiers procédés d'anesthésie par hibernation pour opérer les blessés de guerre. Il se distingua avec la synthèse des premiers antidépresseurs utilisés en neuropsychiatrie dès les années 1950. La connaissance précise du fonctionnement du système neurocérébral constitue la base d'une réflexion épistémologique et philosophique sur le comportement des hommes en société. Loin d'en rester à l'analyse des seuls processus biologiques, cet auteur a pratiqué et promu une approche transdisciplinaire en s'appuyant sur les théories cybernétiques et systémiques pour explorer l'ensemble des régulations à chacun des niveaux d'organisations de la matière et surtout du vivant. C'est à l'occasion de la création d'un enseignement au Centre universitaire expérimental de Vincennes en 1968 sur «Biologie et urbanisme » (1968-1971) qu'il transpose l'approche systémique et la théorie cybernétique au phénomène urbain en tant que système complexe. Son ouvrage compilant les hypothèses et analyses de cette expérience d'interdisciplinarité, L'homme et la ville (Flammarion, 1971), offre une illustration sur la façon dont les sciences du vivant éclairent l'organisation politique des sociétés humaines.
\end{abstract}

\begin{abstract}
Man and the City, a systems approach to urban planning by neurobiologist Henri Laborit. Neurobiologist Henri Laborit (1914-1995), a renowned French researcher and philosopher, first developed anesthetic methods to artificially hibernate wounded soldiers being operated. He distinguished himself with his work on the synthesis of the first antidepressants used in neuropsychiatry in the 1950ies. In-depth understanding of the neuro-cerebral system and its implications for the comportment of animals and humans led Laborit to develop a global and interdisciplinary reflection on human comportment in society. Beyond the analysis of biological and neurophysiological aspects, Laborit promoted and applied a veritable interdisciplinary approach by bringing in cybernetics and systems theories to explore the regulations at work at each level of biological and sociological organizations. The seminar « Biology and urbanism ", held between 1968 and 1971 at the Paris-Vincennes Experimental Faculty, is based on this cybernetics and systems approach applied to the dynamics of urban systems. His book L'homme et la ville (Man and the City, Flammarion, 1971) illustrates this epistemological posture by which the life sciences shed light on the political organization of human societies.
\end{abstract}

Henri Laborit fait partie d'une génération de penseurs convaincus de la nécessité d'une approche transdisciplinaire pour apporter des réponses aux enjeux de la société contemporaine. C'est à l'issue d'un colloque entre biologistes, sociologues et philosophes que Robert Buron, Edgar Morin, Jacques Robin et Henri Laborit décidèrent de créer en 1966 un groupe de réflexion qui prit le nom de Groupe des dix (Robin, 2007). Ces quatre pionniers furent rapidement rejoints par Jacques Attali, René Passet et Joël de Rosnay. L'objectif de ce groupe consistait à mieux comprendre et cerner les rapports entre les sciences et les techniques, d'un côté, la culture et le politique, de l'autre. Ce club de réflexion, animé par Jacques Robin, a rassemblé d'autres penseurs tels que Michel Serres, Michel Rocard, Jacques Delors, Henri Atlan, Francisco Varela. Tous, à partir de leurs disciplines respectives,

Auteur correspondant : cyrille.harpet@ehesp.fr

Cyrille Harpet est chercheur associé à l'UMR 5600 EVS (Labex IMU), à Lyon. 
cherchaient à féconder mutuellement leurs recherches, en quête d'un nouveau paradigme.

La place occupée par Henri Laborit dès la formation du Groupe des dix est celui d'un neurobiologiste de renom ayant prolongé ses travaux de recherche sur les mécanismes cérébraux par une relecture systématique et scrupuleuse des comportements humains et sociaux à partir d'une grille d'analyse de la cybernétique et de la théorie de l'information. En cela, il a œuvré pour la compréhension des systèmes complexes et de la dynamique des structures à chacun des niveaux d'organisation du vivant (de la cellule aux écosystèmes).

Lors de la création du Centre universitaire expérimental de Vincennes en 1968, né d'un épisode de renversement des schémas d'organisation traditionnels des universités, Henri Laborit a été sollicité par les étudiants pour conduire une expérience pédagogique innovante. Il s'agissait d'une unité de valeur - on parlerait aujourd'hui d'unité d'enseignement - « Biologie et urbanisme ", laquelle deviendra par la suite une unité de recherche de troisième cycle. Durant trois années (19681971), le neurobiologiste a tenté « d'apporter la connaissance générale actuelle des plans d'organisation sousjacents (à ceux du niveau sociologique), de la molécule aux comportements ${ }^{1} \gg$ (p. 9, introduction), dont les grandes lignes ont ensuite été transcrites dans son ouvrage L'homme et la ville (Laborit, 1971). L'intérêt de ce livre est d'être l'illustration notable d'un essai de transdisciplinarité, lequel a été suscité par le Groupe des dix et devant fournir les éléments d'une méthodologie d'appréhension nouvelle du problème urbain.

Renonçant à la fonction d'enseignant, et sortant de l'académisme classique, Henri Laborit a voulu conduire cette expérimentation sous la forme d'une recherche participative, "d'une recherche de groupe, d'un essai de compréhension d'un système complexe » (p. 10, introduction). En somme, en parallèle au Groupe des dix et hors du cadre du laboratoire d'eutonologie $\mathrm{e}^{2} \mathrm{qu}^{\text {ill a fondé }}$ à l'hôpital Boucicaut à Paris, il a animé, une réflexion - plus qu'un enseignement - auprès d'étudiants urbanistes, pour appliquer la dynamique cybernétique à l'échelle des phénomènes urbains.

Cet exercice auquel s'est prêté Henri Laborit entre 1968 et 1971 nous retiendra dans cet article pour illustrer, à travers la question des "mondes urbains ", l'approche transdisciplinaire promue et expérimentée à Vincennes. La première partie souligne quelques points de convergence conceptuels entre les membres fondateurs du Groupe des dix. La deuxième partie porte sur les prérequis scientifiques établis par Henri Laborit quant à

\footnotetext{
1 Sauf mention contraire, toutes les citations dans cet article sont issues de l'ouvrage d'Henri Laborit, L'homme et la ville (1971, Paris, Flammarion).

2 En physiologie, étude du maintien d'un tonus normal.
}

la compréhension des comportements sociaux et du phénomène de l'urbanisation. La connaissance du système neurocérébral constitue le fondement scientifique d'analyse des comportements humains. La troisième partie traite, à travers l'histoire, du phénomène d'urbanisation, dont l'essor et l'expansion signalent, pour l'auteur de L'homme et la ville, la persistance de rapports de domination et de ségrégations entre catégories sociales et économiques. Ce chapitre ouvre la perspective d'une approche renouvelée de l'urbanisme en considérant les capacités intégratives et systémiques des disciplines. La ville comme système régulé en tendance ${ }^{3}$ peut faire l'objet d'une reconversion de sa conception à son organisation si l'ensemble des enjeux politiques, écologiques et de santé publique sont pris en considération. La nécessaire interdisciplinarité pour concevoir des systèmes complexes tels que ceux urbains et environnementaux est sans cesse invoquée par le Groupe des dix, sans avoir trouvé encore sa pleine effectivité. Les problématiques de développement durable, à l'échelle globale puis aux divers échelons locaux, ont permis une nette avancée de cette appréhension transversale des enjeux, d'une vision intégrée des risques environnementaux et, plus largement, de société. La rationalité classique aura procédé par un cloisonnement disciplinaire que les membres du Groupe des dix auront sérieusement bouleversé. Laborit rejoint ces promoteurs de l'interdisciplinarité en y ajoutant sa revendication d'un développement de l'homme imaginant, c'est-à-dire d'un homme pour qui l'imaginaire constitue une capacité à explorer et développer, en liant des niveaux d'organisation jusque-là tenus pour dissociés et sans interactions. Son propos et sa méthodologie permettent d'inscrire l'évolution urbaine dans une vision biopolitique où l'homme devient autant effecteur d'un système organisé que pris dans des régulations complexes. C'est quasiment vers une "écologie de l'esprit » (Bateson, 1972) que nous oriente son œuvre plus que vers une écologie urbaine humaine.

\section{Trois points de convergence entre les membres du Groupe des dix}

Le Groupe des dix mérite un bref rappel des points de convergence conceptuelle et des affinités théoriques qui lient ses membres. Certes, tous partagent une curiosité

3 Dans le langage de la cybernétique, dans la théorie systémique, et en physiologie, le concept de rétroaction (feed-back) est fondamental : il désigne une " action en retour " d'un effet sur sa propre cause, action déclenchée par les variations de facteurs à l'origine de cette action. On distingue les rétroactions négatives qui correspondent à un fonctionnement en constance, c'est-à-dire à des réponses atténuées par la boucle des réponses, et les rétroactions positives qui correspondent à un fonctionnement en tendance (avec amplification des réponses). 
intarissable pour les sciences, un esprit de questionnement intempestif sur les choix de société, un goût pour «la reliance entre les savoirs disciplinaires», un irrépressible besoin de "disséminer ses connaissances" pour engager des transformations et « changer d'ère ${ }^{4}$ ». Mais on peut aussi retenir trois faits de connivence intellectuelle, signifiant les affinités scientifiques établies entre ses membres autour d'un nouveau paradigme.

En premier lieu, nous retrouvons une démarche à laquelle chacun des chercheurs du Groupe des dix s'est initié, partant d'une spécialité et d'un champ disciplinaire spécifique pour s'en extraire et en élargir le cadre $\mathrm{d}$ 'analyse du phénomène humain. Chacun s'en est remis à un cadre théorique commun, celui de la théorie de l'information, de la cybernétique et de la systémique.

En second lieu, le paradoxe des systèmes autoréférentiels a été soulevé par la majorité de ces auteurs, se fondant chacun sur l'analyse fournie par Francisco Varela (Dupuy, 1982, p. 122). Dans un travail algébrique, celui-ci a dégagé les propriétés des comportements propres valables pour une large catégorie de systèmes autoréférentiels. L'autoréférence introduit une relation circulaire entre deux niveaux de logique différents, celui du métalangage et celui du langage objet. Chacun des membres du Groupe des dix en est devenu à son tour un énonciateur, avec Francisco Varela considérant ce paradoxe comme principe de la vie et de la conscience de soi, Henri Atlan comme principe de la dynamique du vivant à l'échelon biologique (le vivant qui s'observe luimême, le cerveau qui écrit la théorie du cerveau...), Edgar Morin à travers l'ensemble des systèmes biologiques, psychologiques, sociaux... jusqu'à Cornélius Castoriadis qui décèle les extériorités signifiantes garantissant l'organisation sociale à travers l'histoire des sociétés, formes de pseudo-transcendances secrétées par l'homme puis désacralisées : nature, raison, histoire, État... (cf. notamment ses ouvrages L'institution imaginaire de la société et Les carrefours du labyrinthe). Henri Laborit rejoint ces énonciations du paradoxe de l'autoréférence à travers l'examen des mécanismes de maintien des structures du vivant. Les conditions de ce maintien seraient «la quantité d'énergie et d'information nécessaire au maintien d'une structure », lesquelles définissent biologiquement et thermodynamiquement un besoin, selon l'auteur (p. 26).

«Dans cette définition entrent les besoins instinctuels, ceux nécessaires au maintien de structures innées, et régis, nous le verrons, par le cerveau reptilien, l'hypothalamus en particulier. Entrent aussi dans cette définition les besoins résultant des automatismes créés par l'apprentissage et commandés par la mémoire, les habitudes et le système limbique. » (p. 26)

4 Selon les mots d'Edgar Morin, dans Le Monde du 18 août 2007, en hommage à Jacques Robin, décédé le 7 juillet 2007.
Comment - en troisième et dernier lieu - sortir de l'autoréférence biologiquement programmée et quasi déterminée par la structure neurocérébrale dans ses soubassements les plus archaïques? Parmi les systèmes hétéroréférentiels, nous pouvons citer l'ensemble des objets constitués par les civilisations, les objets techniques, les modes de production en agriculture, les systèmes industriels, la production de connaissances scientifiques, la production artistique, la civilisation urbaine. La ville, dans la vision d'une biopolitique avancée par Laborit, devient « une production de la vie, une sécrétion, une enveloppe », puis, dans une vision techniciste, « un outil et un vêtement, une cuirasse et une limitante, un lieu d'échanges, une membrane» (p. 27). Sans la confondre avec un organisme, la ville devient l'un des moyens de maintien et de contrôle d'une structure sociale, mais aussi un milieu de rapports de force entre groupes humains. Pourtant, elle devrait constituer la résultante d'une organisation évoluée des sociétés humaines, lieu d'émergence des innovations par les capacités associatives du néocortex cérébral. Pour traiter de l'évolution culturelle et technique de l'humanité en toute extériorité et hors du schéma de l'imitation généralisée et aveugle entre les acteurs, il s'agit de se positionner en toute transcendance pour dégager le sens du projet technicien, celui cherchant à s'autonomiser pour mieux décharger l'homme de ses déterminismes et accéder à la liberté de ses choix.

\section{Les prérequis théoriques de L'homme et la ville}

Les ouvrages d'Henri Laborit peuvent se répartir en deux grandes catégories : ceux, issus des travaux de laboratoire ${ }^{5}$, relevant de la spécialité scientifique en neurobiologie et physiologie du système nerveux, et ceux relevant des réflexions philosophiques et destinés au grand public ${ }^{6}$. L'Homme et la ville est un essai de vulgarisation des travaux de biologie et des théories de l'information pour comprendre l'urbanisme sous un nouvel angle. Aussi, depuis son essai Du soleil à l'homme, paru en $1963^{7}$, Laborit s'oblige quasi systématiquement à énoncer de

5 De Physiologie et biologie du système nerveux végétatif au service de la chirurgie de 1950 à Les récepteurs centraux et la transduction des signaux de 1990, en passant entre autres par Réaction organique à l'agression et choc (1952), Résistance et soumission en physio-biologie. L'hibernation artificielle (1954), Bases physio-biologiques et principes généraux de réanimation (1958), Les régulations métaboliques (1965), Biologie et structure (1968), Neurophysiologie. Aspects métaboliques et pharmacologiques (1969), Les comportements. Biologie, physiologie, pharmacologie (1973) et L'inhibition de l'action (1979).

6 Voir la bibliographie détaillée en fin d'article.

7 Il s'agit de son premier ouvrage de vulgarisation scientifique. 
façon pédagogique les bases de connaissance dans trois domaines: la connaissance du système neurocérébral comme prérequis à la compréhension de tout comportement humain, individuel et collectif, dans son fonctionnement biologique et sociologique; les principes et concepts de la théorie des ensembles, de l'information et des systèmes complexes ; enfin, la cybernétique signerait l'aboutissement dans la compréhension de toutes les régulations des systèmes complexes (des machines, des organismes vivants, des milieux écologiques, etc.).

La démarche de Laborit procède ainsi en trois étapes majeures de réflexivité sur la base de l'expérimentation en neurophysiologie, puis sur la base des théories émergentes des systèmes complexes (théorie de l'information, théorie cybernétique et théorie systémique) pour mieux formaliser les interactions entre niveaux d'organisation, du biologique à l'écologique, enfin sur la base de l'analyse des comportements sociaux guidés par des choix biopolitiques sous-jacents.

L'homme et la ville s'ouvre sur un ABC de cybernétique pour expliciter les concepts indispensables à la démonstration suivante : la ville constitue un "produit d'une structure vivante», «un produit social», un moyen au service d'une finalité propre à la société humaine, et donc constitue un " effecteur » dans le jargon cybernéticien, puisque la ville « agit en maintenant la structure du groupe humain », ce dernier devenant un «facteur» de la ville. C'est la rétroaction de l'effecteur «ville» sur le facteur "société humaine» qui constitue donc l'enjeu dans l'approche des systèmes et des niveaux d'organisation (p. 22-23). L'élément essentiel de cette analyse est que l'objet «ville » n'est pas l'objet de motivation, la finalité poursuivie par le groupe humain; il est seulement un moyen indirect pour ce groupe de conserver sa structure, qui est sa seule finalité. La ville devient, sous la plume d'Henri Laborit, un milieu transformé et donc mis en forme, matériellement, mais aussi et surtout socialement, de façon à atteindre un nouveau niveau d'organisation, celui d'un entrelacs de relations plus complexes encore. Notre cybernéticien ne cache plus, à l'occasion de cette analyse de l'évolution du niveau d'organisation que constitue l'échelle urbaine, un certain déterminisme des formes vivantes émergentes et des structures, écartant l'idée de l'aléatoire soutenue par Jacques Monod à cette époque (dans Le hasard et la nécessité). Selon lui, le déterminisme se détecte dans les régulations des niveaux susjacents et sous-jacents. Le milieu urbain auquel se réfère Laborit est entre deux niveaux d'organisation, celui des comportements biologiquement induits dans les communautés humaines et celui des interactions avec l'environnement physique ou écologique. Son œuvre contribue à un recentrage des sciences sur le monde du vivant, et non plus sur les références traditionnelles de la physique. Ce ne sont plus les lois fondamentales de la matière et de ses transformations qui ont guidé l'édification des cités humaines, pour en constituer des architectures spécifiques selon les époques et les cultures. Ce ne sont plus seulement les lois écologiques des milieux naturels qui ont dirigé l'installation des groupes humains sur la surface de la planète, mais ce sont aussi les capacités de nouvelles formations sociales, explorant de multiples combinaisons sociologiques, pareilles à des colonies cellulaires, à des communautés d'organismes unicellulaires et pluricellulaires. De même, le concept de sélection dans l'évolution des espèces ne prédomine plus dans cette vision renouvelée, ce sont ceux « d'addition de formes existantes, de combinaison, de symbiose, d'hybridation » (p. 37) qui prévalent. La civilisation urbaine devient ainsi un processus de complexification à un niveau d'organisation supérieur (non hiérarchiquement, mais structurellement), sur un mode conservateur des plus anciennes formes biologiques et sociales.

Ce recentrage biologique trouve sa pleine expression dans le rappel persistant de la structure cérébrale, autrement dit en s'adossant aux trois cerveaux de Mc Lean (1970-1978). La tripartition cérébrale (cerveau reptilien, système limbique et néocortex) est décrite par Laborit de façon à réinscrire les comportements humains dans des schémas d'automatisme qui en déterminent les motivations. De là, les éléments de structure des comportements à travers le filtre biologique servent de base à la compréhension des autres niveaux d'organisation, notamment ceux formant des systèmes complexes tels que les comportements sociaux.

La théorie de l'information et celle de la cybernétique apportent le cadre conceptuel permettant d'analyser de manière transversale et interdisciplinaire les interactions entre ces niveaux d'organisations du vivant. Dès 1950, Laborit explore l'œuvre des théoriciens de la cybernétique $^{8}$, science récente forgée au carrefour des mathématiques et de la neurophysiologie. Le savant mathématicien Norbert Wiener devait étudier, à la demande du gouvernement américain, les possibilités de réglage automatique de tirs de "défense contre les aéronefs" (DCA) pour prévoir et intercepter les trajectoires des avions ennemis. Il se tournera du côté des neurophysiologistes (Rosenblueth, Mc Culloch, Lorente de Nô) pour définir des mécanismes communs de régulations et de contrôles dans les systèmes de communication du vivant ou des machines. Dès 1948, Wiener choisit le terme de

\footnotetext{
8 Suite à la première conférence de 1942, sont publiés en 1943 les deux articles fondateurs de la cybernétique : «Behavior, purpose and teleology » dans lequel Arturo Rosenblueth, Norbert Wiener et Julian Bigelow étudient les modèles d'organisation sous-jacents aux comportements finalisés et «A logical calculus of ideas immanent in nervous activity", dans lequel Warren Mc Culloch et Walter Pitts étudient les modèles d'organisation sous-jacents à la perception.
} 
« cybernétique » pour désigner cette "science qui étudie exclusivement les communications et leurs régulations dans les systèmes naturels et artificiels ». Ce sont les notions de feed-back (rétroaction) et de système régulé qui enthousiasment Laborit, au point d'en chercher un peu partout dans l'organisme. Il faut reconnaître une orientation commune à Laborit et à certains membres du Groupe des dix, portant spécifiquement sur cette théorie cybernétique, puisqu'autant De Rosnay, Morin, Atlan, Dupuy que Passet et Robin y font référence pour la modélisation des systèmes d'information qu'elle autorise. La cybernétique présente pour Laborit, qui s'y réfère systématiquement dans l'ensemble de ses ouvrages de réflexion, l'intérêt de configurer des ensembles d'éléments relevant de niveaux d'organisation distincts mais tous reliés et en interactions. Laborit sera le premier à oser ainsi parcourir les niveaux d'organisation de la molécule à la biosphère. Cela lui sera souvent reproché par la suite comme étant une vision de généraliste empreinte d'extrapolations et d'un certain réductionnisme. Il sera ainsi tenté de généraliser les lois de la biologie au politique et au social.

« La relation à l'écosystème constitue le troisième volet de sa démarche. La molécule active, la cellule, le tissu, l'organe, le corps, ne sont jamais séparés de leur environnement immédiat, de leur écosystème microscopique ou macroscopique : ils s'intègrent dans un tout, lui-même ouvert sur un environnement plus vaste encore. Cette vision amène Laborit à quitter la biologie, au sens "disciplinaire" du terme pour s'intéresser à l'environnement humain et ses corollaires économiques et politiques. » (De Rosnay, 1995)

C'est en partie la ville qui va constituer un environnement propice à l'analyse des interactions biologiques et sociologiques, dans le sens où le système urbain permet une vision historique des civilisations (Lewis Mumford [1961] en est l'une des références), où les interactions entre organismes et le milieu de vie sont fortes (l'écosystème urbain), enfin, où les rapports politiques sont les plus perceptibles (une biopolitique urbaine).

Comme nous l'avons dit, en 1968, au cœur du mouvement de contestation, Laborit est sollicité par des étudiants en urbanisme de l'université de Vincennes pour enseigner la biologie des comportements avec la conception urbaine, architecturale et l'habitat. C'est l'ouvrage Biologie et structure (1968) qui a suscité cet intérêt, et l'expérience s'avère rapidement un succès. D'emblée, Laborit s'interroge :

«L'urbanisme contemporain peut aussi se concevoir sous une forme expérimentale : faisons une ville et nous verrons bien ce qui $\mathrm{s}^{\prime} \mathrm{y}$ passe. Mais est-ce là l'expérimentation? N'est-ce pas plutôt un jeu de pile ou face? Une œuvre humaine comme la cité peut-elle s'expérimenter dans l'ignorance des mécanismes qui dirigent le comportement de son ouvrier, l'homme, ou plus exactement du groupe humain qui la conçoit et la réalise ?» (p. 10)

\section{L'urbanisation comme processus de maintien des rapports sociaux de domination}

Le phénomène urbain constitue « un moyen secondaire pour l'homme en société de maintenir sa structure» (p. 22), autrement dit une production accessoire issue de l'évolution qui ne contribue qu'indirectement au maintien d'une structure sociale. La finalité n'est donc pas de construire une ville, selon Laborit, ce qui met de côté toute orientation esthétique de l'architecture, mais elle serait, à l'échelle d'une société, de maintenir des relations de domination. La lecture marxiste de l'auteur est renforcée par le renvoi systématique à la société bourgeoise, laquelle est portée par l'accumulation de capitaux et obnubilée par le profit (sous-entendu marchand). Il est clair que la finalité de l'objet ville (équivalent d'une production ou d'une fabrication technique) ne peut être programmée dans la structure de la ville, aussi complexe soit-elle, mais qu'elle est fixée et imposée par l'homme.

Laborit réfute catégoriquement l'analogie entre organisme biologique et cité humaine sur le plan fonctionnel, car le changement de niveau d'organisation ne permet pas de conserver les mêmes lois au niveau biologique et au niveau sociologique. Il est vrai que l'analogie entre l'espace urbain et l'espace corporel a persisté dans la conception occidentale, comme si, du corps à la cité humaine, les modèles se rejoignaient et nouaient une étrange parenté. L'auteur de L'homme et la ville qualifie la ville comme l'une des productions humaines, une "sécrétion », « une enveloppe » (p. 27) mais l'analyse comme un phénomène social (« une production sociale ») en attribuant à la sémantique et, plus généralement, au langage, la fonction d'alibis de déterminismes biologiques inconscients. La ville est le lieu des formes instituées de sociabilité, lesquelles sont toutefois révélatrices de rapports de domination entre races, classes et catégories sociales.

Cela n'est pas sans rappeler l'analyse des jeux de pouvoir que Lewis Mumford avait déjà spécifiquement fait valoir dans son ouvrage The city in history. Its origins, its transformations, and its prospects (1961). Henri Laborit conforte cette thèse par le recours aux automatismes biologiques qui détermineraient cette configuration de centralisation des pouvoirs par des minorités (la bourgeoisie dans la terminologie marxiste reprise par Laborit). La ville constitue un moyen et une production humaine, une sorte de « sécrétion » qui dissimulerait tant bien que mal les tendances dominatrices à l'échelle des espaces urbains et des organisations des groupes humains. La ville forme un système complexe concentrant une série de facteurs, allant du biologique au politique, en passant par les échanges économiques généralisés, exerçant des effets avec régulation en tendance sur les groupes humains. Les derniers facteurs à considérer dans un tel 
système urbain seraient ceux d'ordre écologique et sanitaire, cités rapidement par Laborit, pour lesquels les effets sont encore insuffisamment analysés et observés. Nous en expliciterons les prémices en matière d'urbanisme et de santé publique.

Le milieu urbain constitue dans l'ouvrage de Laborit une « niche environnementale » particulière, non pas au sens écologique du terme comme on aurait pu s'y attendre, mais en tant que « structure sociale » (p. 55). Et cet environnement urbain, cette urbanisation, insiste l'auteur, ne stimule pas les capacités associatives du néocortex, du cerveau imaginant, mais réactive la série de comportements d'agression. Il se base sur des travaux d'éthologues, dont ceux, fondateurs, de Konrad Lorenz (1969) et Robert Ardrey (1967), qui montrent que le confinement accroît considérablement l'agressivité en raison des contraintes imposées aux réponses face aux besoins primaires (ressources, conjoints pour la reproduction, et territoires). C'est à Sally Carrighar (1971) que Laborit s'en remet pour étayer son analyse et conforter l'idée selon laquelle l'urbanisation accentue le phénomène d'inhibition de l'action. La ville rendrait les mesures d'évitement mutuel plus difficiles entre les individus (la ville est présentée comme un espace confiné). En outre, la ville déploie les capacités d'accroissement du profit et de « consolidation de la structure socio-économique ou de la classe qui initie la ville» (p. 23). Cela a pour conséquence une agressivité détournée vers d'autres alibis, ceux de rapport de domination entre individus et groupes sociaux. De nouvelles formes de dépendance se sont instaurées au cours de la civilisation urbaine, avec la spécialisation des tâches et des fonctions, l'instauration d'un « système d'échanges généralisés » (Mandel, 1962). Le passage d'une structure sociale archaïque sans spécialités à une organisation hiérarchisée et basée sur l'addition de spécialistes dont certains détiennent la force et/ou les moyens de production, et/ou le capital et la plus-value, rejoindrait un certain déterminisme déjà observé dans l'évolution du vivant, celui guidé par « un besoin de domination paléocéphalique » (p. 76, L'homme et la ville). La ville constitue un milieu propice et organisé pour la grande production, promotion puis diffusion essentiellement de biens de consommation, de marchandises, sous couvert d'un mythe de satisfaction de besoins et $\mathrm{d}$ 'accès au bonheur de tous. Il en va ainsi de formes d'aliénations à des objets et propriétés issus de la société technique et industrielle, entretenues par un mythe qui cache mal ou si peu les déterminismes dont chacun demeure inconscient. Les rapports de domination que souligne Laborit se réfèrent à une analyse marxiste quant à l'exploitation de la force de travail d'une majorité par une minorité, sous forme de lutte de classes, mais au sein desquelles l'aliénation est tout aussi présente, «le prolétaire comme le capitaliste est exploité par un mythe, ou plus exactement par un déterminisme dont ils sont inconscients l'un et l'autre » (p. 80). Pour l'espace proprement urbain, ce sont les formes de ségrégations spatiales qui traduisent ces rapports de domination. La structure de ville influe dès lors sur la structuration des groupes humains, la ville constituant un «effecteur" opérant une rétroaction ici positive (donc «en tendance ", appelée aussi " pompage » en langage cybernétique), puisque dans les sociétés marchandes, « la structure ségrégative du groupe humain sera elle-même aggravée, et une telle aggravation peut conduire à la révolte du groupe humain » (p. 23). L'urbanisme est ici revu sous l'angle sociologique et sous l'angle des interactions socioéconomiques entre les acteurs. Les régulations en tendance s'exercent autant sur le plan écologique des ressources et des milieux (boucle positive $\mathrm{d}^{\prime}$ accroissement de prélèvement des ressources, d'occupation des milieux, de pollutions et nuisances, de ce qui est aujourd'hui nommé «empreinte écologique») que sur le plan d'aménagement de l'espace et de l'organisation sociologique des groupes humains. Les interactions directement biologiques entre l'homme et la ville (intensité lumineuse, hygrométrie, espace, volume, etc.) ne sont pas abordées par Laborit, laissant ces aspects aux spécialistes de l'hygiène urbaine « telle qu'elle est classiquement traitée » (p. 26). Il ne traite pas non plus des impacts biologiques relatifs à l'ensemble des facteurs écologiques des éléments tels que l'eau, l'air, les sols, les microorganismes. Or c'est ce champ de l'épidémiologie (écologique notamment) qui aujourd'hui est investi par la recherche en santé publique et en santé environnement, à travers l'analyse fine de la multitude des agents dits xénobiotiques (physiques, chimiques, biologiques) et des expositions des populations. Cela est d'autant plus étonnant de voir Laborit préconiser une approche globale, dynamique et relationnelle du phénomène urbain qu'il n'aborde pas ces effets biologiques et épidémiologiques faisant l'objet d'analyses de risques devenues préoccupantes (tels que les perturbateurs endocriniens disséminés dans les milieux de vie). Laborit a maintenu sa vision de l'individu biologique à travers la détermination neurophysiologique qu'il inscrit jusque dans les rapports sociaux. Mais il n'aura pas eu le temps, sans doute, de considérer les facteurs d'influence biologique et neurophysiologique que constituent les substances et molécules disséminées dans les milieux anthropisés. Ce ne sont plus seulement les rapports sociaux qui transforment les individus, mais aussi l'environnement écologique (celui d'une écologie urbaine, par exemple), l'environnement technique (lié au monde industrialisé), facteurs externes de la structure sociale, variables selon les lieux et les époques. Pour Laborit, « le facteur principal est interne, il réside dans la structure du système nerveux humain, et dans la connaissance et donc dans la conscience que nous pouvons en avoir» (p. 97). Le système urbain est considéré par Laborit comme clos dans 
ses régulations, à l'échelle de l'évolution depuis le néolithique, avec une invariance de structure qui est celle de la biologie stable des comportements humains qui animent le groupe social. Seul «le cerveau imaginant constitue le servomécanisme qui permettrait de sortir du feed-back infernal de la production pour le profit, et du profit pour la domination»(p. 102). C'est seulement lorsque la ville ne sera plus conçue comme système technique et donc comme un simple moyen de maintien des structures sociales du pouvoir qu'une nouvelle harmonie entre les hommes, entre les hommes et leur milieu de vie, trouvera sa pleine expression, dans la pleine conscience des motivations instinctives et des automatismes imposés par la forme sociale. L'investigation scientifique prônée doit porter sur un environnement devenu un laboratoire (l'écosystème) dégagé des préjugés ancestraux (une "bonne nature», un "méchant humain ») et considérer l'urbanisation comme un ensemble englobant l'urbanisme. L'urbanisation serait le processus global relevant des problèmes d'écologie générale (de préservation des écosystèmes planétaires), l'urbanisme relevant du niveau de l'écologie locale (p. 111). Sur chacun de ces niveaux, l'auteur invoque la nécessité d'une recherche scientifique interdisciplinaire, et non plus seulement des chaînes de causalité linéaire, «mais de façon beaucoup plus complexe par des systèmes de corrélations hiérarchisées » (p. 117), et réclamant la construction de modèles. Nous sommes en 1971, et Laborit est déjà au fait des travaux scientifiques relatifs à la concentration en dioxyde de carbone dans l'atmosphère et des risques de changements climatiques globaux. Pour ce qui est de l'écologie urbaine, l'auteur s'en remet non seulement à son analyse des déterminismes comportementaux, mais aussi et surtout à la critique de l'uniformisation généralisée des conditions de vie. Il en appelle alors à René Dubos, autre prix Lasker, qui, lors d'un discours à l'Unesco en 1966 déclare :

\footnotetext{
« il nous faut fuir autant l'uniformité de notre environnement que la conformité totale en matière de comportement et de goûts. Nous devons au contraire chercher à diversifier autant que possible les milieux où nous vivons. La richesse et la diversité des milieux physiques et sociaux constituent un aspect essentiel du fonctionnalisme, que ce soit en matière de planification des zones rurales et urbaines, de conception de l'habitat ou de l'aménagement de la vie privée. » (p. 122)
}

Aux tendances à la concentration des puissances (médiatiques, marchandes, financières, techniques et industrielles), d'expansion des métropoles que recèle le phénomène d'urbanisation, Laborit, reprenant Dubos, oppose les capacités de diversification et d'associations créatrices des communautés humaines qui trouvent dans une finalité autre que celle du système, un servomécanisme en contrôle par des régulations en constance. Il s'agit d'une révolution interne à l'homme, soit par une phase de crise de conscience partagée (révolution intellectuelle), soit sous la pression de la nécessité de survie immédiate (révolution biologique). La connaissance de ces deux facteurs de changement et de leurs mécanismes permettrait d'éviter de reconduire les mêmes erreurs, d'éviter le pire et de porter une nouvelle utopie, ici une utopie urbaine sans doute, mais à l'échelle d'un processus planétaire (p. 114).

\section{Conclusion}

Parmi les membres du Groupe des dix, Laborit occupe une place spécifique par un parcours de recherche scientifique véritablement transversal et interdisciplinaire, fécond depuis l'expérimentation neurobiologique des organismes vivants jusqu'à une réflexion sur l'évolution de l'humanité à l'échelle cosmique. Dans cette épopée anthropologique, il reste le seul à organiser ses connaissances au profit d'une nouvelle expérimentation, celle de l'analyse biopolitique du phénomène d'urbanisation.

"Ainsi dans "L'homme et la ville", Laborit intègre et décline sa vision de l'être biologique en relation avec son écosystème urbain. Il montre avant beaucoup d'auteurs les limites du système économique fondé sur la croissance, le gaspillage des ressources naturelles et la création des exclusions. Sa vision prophétique des années 1960 a été progressivement confirmée. Les grandes villes sont devenues le point de convergence des principaux problèmes que l'humanité devra aborder au tournant du millénaire. Sa vision systémique a inspiré de nombreux architectes, urbanologues, sociologues concernés par les villes du futur. » (De Rosnay, 1995)

Laborit a revendiqué cette interdisciplinarité pour concevoir des systèmes complexes telle que la ville qui constitue un fait sociologique majeur dans l'évolution humaine. Somme toute, l'exercice est très audacieux, et difficile pour qui doit franchir les frontières scientifiques afin de dégager une vue d'ensemble et les principes de compréhension. Considérer la multitude des régulations qui gouvernent les divers niveaux d'organisation peut retarder, voire empêcher, la décision. Les anciens élèves du séminaire de Vincennes reconnaissent «que l'interprétation des comportements, telle qu'elle leur a été transmise par Laborit, entre en compte dans leurs conceptions de la ville et de l'habitat » (Laborit, 1982). Mais la limite en est, malgré l'influence exercée, le refus par l'auteur de L'homme et la ville d'une application directe de la biologie à l'urbanisme, et donc à l'aménagement de l'espace. Le contexte des années 1970 était à l'engouement pour une nouvelle vision de l'homme, pacifique, imaginatif, créatif, désintéressé, débarrassé des préjugés moraux hérités. En libérant l'imaginaire et en justifiant l'imagination au pouvoir, Laborit ouvre la 
perspective d'une révolution intellectuelle sur la base tangible des preuves biologiques plutôt que sur la phraséologie de la psychanalyse. Ses ouvrages suivent de très près l'actualité scientifique dans moult domaines, et, en cela, il excelle par les références choisies et la traduction dans un langage accessible qu'il veut constituer en langage commun, celui de l'unification de l'espèce humaine. L'expérimentation urbanistique requiert une vision large et globale, mais surtout un temps long, celui de l'édification pour des générations. Former les praticiens de l'urbanisme est une chose, leur faire concevoir l'urbanisation sur un autre plan que celui lié au maintien des rapports sociaux de domination est d'une autre ampleur et un nouveau pari, au regard de la complexité des facteurs qui en gouvernent l'effectivité. C'est à ce titre que la révolution neurobiologique appelée par l'auteur de L'homme et la ville, ouvrant sur un imaginaire passant d'une écologie urbaine vers une écologie de l'esprit urbain, ne saurait suffire. Il y manque ce que Jacques Robin appelle la multidimensionnalité de l'esprit, non celle du cerveau. «Si Laborit reconnaît bien les différents niveaux de complexité du réel, il ne comprend pas qu'il est aussi multidimensionnel, qu'il y a différents plans, différents points de vue sur la réalité, pas seulement des niveaux d'organisation. Le réel est irrémédiablement "pluriel" » (Zin, 2001). Celui de la cité humaine urbaine aussi. En effet, au-delà des strates de l'édification urbaine (par niveaux d'organisation), une anthropologie appréhendera le phénomène urbain en tant que fait social total, par l'usage des représentations, des perceptions et des symboles qui en diffractent les lectures.

\section{Remerciements}

L'auteur remercie Henry Dicks (Université Lyon 3Jean Moulin) pour ses conseils avisés lors de la rédaction de ce texte.

\section{Références}

Ardrey, R., 1967. Le territoire, Paris, Stock.

Bateson, G., 1972. Steps to an ecology of mind. Collected essays in anthropology, psychiatry, evolution, and epistemology, Chicago, University of Chicago Press [Trad. fr. : Bateson, G., 19771980. Vers une écologie de l'esprit, Paris, Le Seuil, t. I : 1977, t. II : 1980].

Carrighar, S., 1971. La guerre n'est pas dans nos gènes, Dialogue, 2, I, 23-34.

Castoriadis, C., 1975. L'institution imaginaire de la société, Paris, Le Seuil.

Castoriadis, C., 1978. Les carrefours du labyrinthe, Paris, Le Seuil.

Dubos, R., 1966. Man and his environment: Biomedical knowledge and social action. PAHO/WHO, scientific lectures, 131, Washington (DC), Pan American Health Organization.
Dupuy, J.P., 1982. Ordres et désordres, Paris, Le Seuil.

Laborit, H., 1950. Physiologie et biologie du système nerveux végétatif au service de la chirurgie, Paris, G. Doin et Cie.

Laborit, H., 1952. Réaction organique à l'agression et choc, Paris, Masson et Cie Éditeurs.

Laborit, H., 1954. Résistance et soumission en physio-biologie. L'hibernation artificielle, Paris, Masson et Cie Éditeurs.

Laborit, H., 1958. Bases physio-biologiques et principes généraux de réanimation, Paris, Masson et Cie Éditeurs.

Laborit H., 1959. Les destins de la vie et de l'homme. Controverses par lettres avec P. Morand sur les thèmes biologiques, Paris, Masson et Cie.

Laborit, H., 1963. Du soleil à l'homme, Paris, Masson et Cie.

Laborit, H., 1965. Les régulations métaboliques, Paris, Masson et Cie.

Laborit, H., 1968. Biologie et structure, Paris, Gallimard.

Laborit, H., 1969. Neurophysiologie. Aspects métaboliques et pharmacologiques, Paris, Masson et Cie.

Laborit, H., 1970. L'homme imaginant. Essai de biologie politique, Paris, Union générale d'éditions.

Laborit, H., 1970. L'agressivité détournée. Introduction à une biologie $d u$ comportement social, Paris, Union générale d'éditions.

Laborit, H., 1971. L'homme et la ville, Paris, Flammarion.

Laborit, H., 1973. Les comportements. Biologie, physiologie, pharmacologie, Paris, Masson et Cie.

Laborit, H., 1973. La société informationnelle. Idées pour l'autogestion, Paris, Éditions du Cerf.

Laborit, H., 1974. La nouvelle grille, Paris, Robert Laffont.

Laborit, H., 1976. Éloge de la fuite, Paris, Robert Laffont.

Laborit, H., 1978. Discours sans méthode, Paris, Stock.

Laborit, H., 1979. L'inhibition de l'action, Paris, Masson et Cie.

Laborit, H., 1980. Copernic n'y a pas changé grand-chose, Paris, Robert Laffont.

Laborit, H., 1982. L'alchimie de la découverte, Paris, Grasset.

Laborit, H., 1983. La colombe assassinée, Paris, Grasset.

Laborit, H., 1987. Dieu ne joue pas aux dés, Paris, Grasset.

Laborit, H., 1990. Les récepteurs centraux et la transduction des signaux, Paris, Masson.

Laborit, H., 1992. L'esprit du grenier, Paris, Grasset.

Laborit, H., 1992. Étoiles et molécules, Paris, Grasset.

Laborit, H., 1994. Les bases biologiques des comportements sociaux, Anjou (Québec), Éditions Fides.

Laborit, H., 1994. La légende des comportements, Paris, Flammarion.

Laborit, H., 1996. Une vie, derniers entretiens avec Claude Grenié, Paris, Éditions du Félin.

Lorenz, K., 1969. L'agression, Paris, Flammarion.

Mandel, E., 1962. Traité d'économie marxiste, T.1, Paris, Union générale d'éditions.

Mac Culloch, W., Pitts, W., 1943. A logical calculus of ideas immanent in nervous activity, Bulletin of mathematical biophysics, 5, 4, 115-133, http://link.springer.com/article/ 10.1007\%2FBF02478259\#page-1.

Mac Lean, P.D., 1970-78. Les trois cerveaux de l'homme, Paris, Robert Laffont.

Monod, J., 1970. Le hasard et la nécessité, Paris, Le Seuil.

Morin, E., 2007. En hommage à Jacques Robin, décédé le 7 juillet 2007, Le Monde, 18 août. 
Mumford, L., 1961. The city in history. Its origins, its transformations, and its prospects, New York, Harcourt, Brace \& World [Trad. fr. : Mumford, L., 1964. La cité à travers l'histoire, Paris, Le Seuil].

Robin, J., 2007. Du groupe des 10 à Transversales..., revue Transversales Sciences $\mathcal{E}$ culture, février, http://grittransversales.org/article.php3?id_article $=195$.

Rosenblueth, A., Wiener, N., Bigelow, J., 1943. Behavior, purpose and teleology, Philosophy of science, 10, 1, 18-24, http:/ / courses.media.mit.edu/2004spring/mas966/ rosenblueth_1943.pdf.
Rosnay de, J., 1995. Laborit: de la cybernétique à la systémique, Revue de la Cité des Sciences, Le carrefour du futur, regard vers le $3^{e}$ millénaire, 9 juin, http:/ / www.carrefourdu-futur.com/articles/henri-laborit-de-lacybern $\%$ C3\%A9tique-\%C3\%A0-la-syst $\% C 3 \% A 9 m i q u e /$

Wiener, N., 1948. Cybernetics or control and communication in the animal and the machine, Cambridge (MA), MIT Press [Trad. fr. : Wiener, N., 2014. La cybernétique. Information et régulation dans le vivant et la machine, Paris, Le Seuil].

Zin, J., 2001. De la biologie à la société de l'information, 19 août, http://jeanzin.fr/ecorevo/philo/laborit.htm. 